\title{
Publisher Correction: Anderson light localization in biological nanostructures of native silk
}

Seung Ho Choi (1) ${ }^{1}$, Seong-Wan Kim², Zahyun $\mathrm{Ku}^{3}$, Michelle A. Visbal-Onufrak', Seong-Ryul Kim², Kwang-Ho Choi ${ }^{2}$, Hakseok Ko ${ }^{4,5}$, Wonshik Choi ${ }^{4,5}$, Augustine M. Urbas ${ }^{3}$, Tae-Won Goo ${ }^{6}$ \& Young L. Kim ${ }^{1,7,8}$

Correction to: Nature Communications https://doi.org/10.1038/s41467-017-02500-5, published online 31 January 2018

The original PDF version of this Article contained errors in Equations 1 and 2. Both equations omitted all $\Gamma$ terms, and incorrectly read:

$$
\begin{aligned}
I_{m}\left(\mathbf{r}, E_{\mathrm{ex}}, \omega\right) & =\sum_{h=1}^{H} a_{h}\left(\mathbf{r}, E_{\mathrm{ex}}\right) \frac{h\left(\mathbf{r}, E_{\mathrm{ex}}\right) / 2}{i\left(\omega-\omega_{h}\left(\mathbf{r}, E_{\mathrm{ex}}\right)\right)+{ }_{h}\left(\mathbf{r}, E_{\mathrm{ex}}\right) / 2} \\
\min F & =\sqrt{\frac{1}{S} \sum_{S=1}^{S}\left\|I_{e}\left(\omega_{s}\right)-I_{m}\left(\omega_{s}, a_{h}, \omega_{h}, h\right)\right\|^{2}}
\end{aligned}
$$

The correct form of Equations 1 and 2 are:

$$
\begin{aligned}
I_{m}\left(\mathbf{r}, E_{\mathrm{ex}}, \omega\right) & =\sum_{h=1}^{H} a_{h}\left(\mathbf{r}, E_{\mathrm{ex}}\right) \frac{\Gamma_{h}\left(\mathbf{r}, E_{\mathrm{ex}}\right) / 2}{i\left(\omega-\omega_{h}\left(\mathbf{r}, E_{\mathrm{ex}}\right)\right)+\Gamma_{h}\left(\mathbf{r}, E_{\mathrm{ex}}\right) / 2} \\
\min F & =\sqrt{\frac{1}{S} \sum_{S=1}^{S}\left\|I_{e}\left(\omega_{s}\right)-I_{m}\left(\omega_{s}, a_{h}, \omega_{h}, \Gamma_{h}\right)\right\|^{2}}
\end{aligned}
$$

This has been corrected in the PDF version of the Article. The HTML version was correct from the time of publication.

Published online: 19 March 2018

\begin{abstract}
(c) Open Access This article is licensed under a Creative Commons Attribution 4.0 International License, which permits use, sharing, adaptation, distribution and reproduction in any medium or format, as long as you give appropriate credit to the original author(s) and the source, provide a link to the Creative Commons license, and indicate if changes were made. The images or other third party material in this article are included in the article's Creative Commons license, unless indicated otherwise in a credit line to the material. If material is not included in the article's Creative Commons license and your intended use is not permitted by statutory regulation or exceeds the permitted use, you will need to obtain permission directly from the copyright holder. To view a copy of this license, visit http://creativecommons.org/licenses/by/4.0/.
\end{abstract}

(c) The Author(s) 2018

\footnotetext{
${ }^{1}$ Weldon School of Biomedical Engineering, Purdue University, West Lafayette, IN 47907, USA. ${ }^{2}$ Department of Agricultural Biology, National Institute of Agricultural Sciences, Rural Development Administration, Wanju 55365, Republic of Korea. ${ }^{3}$ Materials and Manufacturing Directorate, Air Force Research Laboratory, Wright-Patterson Air Force Base, OH 45433, USA. ${ }^{4}$ Center for Molecular Spectroscopy and Dynamics, Institute for Basic Science, Seoul 02841, Republic of Korea. ${ }^{5}$ Department of Physics, Korea University, Seoul 02841, Republic of Korea. ${ }^{6}$ Department of Biochemistry, School of Medicine, Dongguk University, Gyeongju 38066, Republic of Korea. ${ }^{7}$ Regenstrief Center for Healthcare Engineering, Purdue University, West Lafayette, IN 47907, USA. ${ }^{8}$ Purdue Quantum Center, Purdue University, West Lafayette, IN 47907, USA. These authors contributed equally: Seung Ho Choi, Seong-Wan Kim, Zahyun Ku. Correspondence and requests for materials should be addressed to Y.L.K. (email: youngkim@purdue.edu)
} 